\title{
Missing Holotypes of Names in Plants, Fungi and Algae Published From India
}

\author{
S. Bandyopadhyay ${ }^{1}$, Gopal Krishna ${ }^{1}$, P. Venu ${ }^{2}$ \\ ${ }^{1}$ Central National Herbarium, Botanical Survey of India, P.O. Botanic Garden, Howrah, \\ West Bengal, India \\ ${ }^{2}$ Environment Protection Training and Research Institute, 91/4, Gachibowli, Hyderabad, \\ Telangana, India
}

*Corresponding Author: Gopal Krishna, Central National Herbarium, Botanical Survey of India, P.O. Botanic Garden, Howrah, West Bengal, India.Email: gopal_bsi@yahoo.co.in

Abstract: A list of names of 54 taxa has been provided whose holotypes could not be located in the herbaria of Botanical Survey of India.

Keywords: Botanical Survey of India, Herbaria, Holotype deposition, Verification

\section{INTRODUCTION}

Protologues and type materials provide crucial data in establishing the identity of a given species. In protologues, everything associated with a name including the citing of holotype is given by the author and the name (as a correct name or as a synonym) gets permanently attached with the designated type. The holotype has a central role in fixing the application of the name concerned. The authors examined names of new taxa published from India in four Indian journals, 'Bulletin of the Botanical Survey of India', 'Indian Journal of Forestry', 'Indian Forestor' and 'Journal of Economic and Taxonomic Botany) for the period between 1990 and 2004. The analysis revealed that names of 54 taxa in actuality with no holotypes traceable in the herbaria cited in the protologues. The examined names along with the cited location of the type (which could not be traced) are presented here alphabetically.

1. Adinandra collettiana T.K.Paul, Bull. Bot. Surv. India 35(1-4): 126. 1997 (1993 publ. 1997). (Theaceae)

Type: Myanmar, Tenasserim, Jaepo, 5000' (1520 m), 17.4.1877, George Gallatly 829A (holotype CAL).

2. Amomum deorianum D.P.Dam \& N.Dam, Bull. Bot. Surv. India 34(1-4): 212. 1997 (1992 publ. 1997). (Zingiberaceae)

Type: India, Meghalaya, Jaintia hills, East of Dawki, 22.4.1972, N.C. Deori 51696 (holotype CAL).

3. Antidesma jayasuriyae Chakrab. \& M.Gangop., J. Econ. Taxon. Bot. 24(1): 21. 2000. (Euphorbiaceae)

Type: Sri Lanka, Ratnapura district, Suriyakanda (Kabaragala no. 1), 16 March 1985, Jayasuriya \& Balasubramaniyam 3256 (holotype CAL).

4. Antidesma keralense Chakrab. \& M.Gangop., J. Econ. Taxon. Bot. 24(1): 23. 2000. (Euphorbiaceae)

Type: India, Kerala, Thiruvananthapuram district, way to Chemungi, 19 May 1979, M. Mohanan 61834 (holotype CAL).

5. Armatella balakrishnanii Hosag., J. Econ. Taxon. Bot. 15(1): 196. 1991. (Meliolaceae)

Type: India, Kerala, Idukki, along the road from Painavu to Kulamavu, in the forest, on the leaves of Cinnamomum malabatrum (Burm.f.) Blume, 18 April 1982, V.B. Hosagoudar 72696 (holotype MH). 
6. Armatella cryptocaryae Hosag., J. Econ. Taxon. Bot. 15(1): 198. 1991. (Meliolaceae)

Type: India, Kerala, Pamba, on the leaves of Cryptocarya bourdillonii Gamble, 10 Oct. 1983, V.B. Hosagoudar 78917 (holotype MH).

7. Armatella indica Hosag., J. Econ. Taxon. Bot. 15(1): 199. 1991. (Meliolaceae)

Type: India, Kerala, Idukki, Calvary mount, on the leaves of Cinnamomum malabatrum (Burm.f.) Blume, 5 Oct. 1983, V.B. Hosagoudar 78160 (holotype MH).

8. Armatella phoebecola Hosag., J. Econ. Taxon. Bot. 15(1): 201. 1991. (Meliolaceae)

Type: India, Kerala, Idukki, Meenumkutty, on the leaves of Phoebe lanceolata Nees, 12 Dec. 1982, V.B. Hosagoudar 73698 (holotype MH).

9. Astragalus drasianus H.J.Chowdhery, Uniyal \& Balodi, Bull. Bot. Surv. India 34(1-4): 209. 1997 (1992 publ. 1997). (Leguminosae)

Type: India, Jammu and Kashmir, Ladakh district, ad locum Dras, August 1988, H.J. Chowdhery \& B.P. Uniyal 85804A (holotype CAL).

10. Baccaurea airyshawii Chakrab. \& M.Gangop., J. Econ. Taxon. Bot. 18(2): 419. 1994. (Euphorbiaceae)

Type: Malay Peninsula, Perak, Larut, Sept. 1882, Kunstler 1408 (holotype CAL).

11. Baccaurea bhaswatii Chakrab. \& M.Gangop., J. Econ. Taxon. Bot. 18(2): 420. 1994. (Euphorbiaceae)

Type: Sumatra, Lampongs, 30 Aug. 1880, Forbes 1653, acc. no. 407625 (holotype CAL).

12. Bambusa assamica Barooah \& Borthakur, Indian J. Forest. 24(4): 503. 2002 (2001 publ. 2002). (Poaceae)

Type: India, Assam, Morigaon, Amsoi, 8th February 1997, C. Barooah 2520 (holotype ASSAM).

13. Bambusa barpatharica Borthakur \& Barooah, Indian J. Forest. 24(4): 505. 2002 (2001 publ. 2002). (Poaceae)

Type: India, Assam, Lakhimpur, Barpathar No. 1, 18th August 1996, C. Barooah 2508A (holotype ASSAM).

14. Bambusa garuchokua Barooah \& Borthakur, Indian J. Forest. 24(4): 506. 2002 (2001 publ. 2002). (Poaceae)

Type: India, Assam, Lakhimpur, Barpathar No. 1, 7th July 1996, C. Barooah 2505 (holotype ASSAM).

15. Bambusa rangaensis Borthakur \& Barooah, Indian J. Forest. 24(4): 508. 2002 (2001 publ. 2002) (Poaceae)

Type: India, Assam, Lakimpur, Ranga R.F., 18th August 1996, C. Barooah 2512A (holotype ASSAM).

16. Biophytum congestiflorum Govind., J. Econ. Taxon. Bot. 20(2): 311. 1996. (Oxalidaceae)

Type: India, South India, W. Ghats, Kerala, Peermede, Azhudha, E. Govindarajalu 5597 (holotype CAL).

17. Biophytum longipedunculatum Govind., J. Econ. Taxon. Bot. 20(2): 312. 1996. (Oxalidaceae)

Type: India, Kerala, Chalakudi Division, Anakayam, E. Govindarajalu 2955 (holotype CAL).

18. Calamus lacciferus Lakshmana \& Renuka, J. Econ. Taxon. Bot. 14(3): 707. 1990. (Arecaceae)

Type: India, Karnataka, evergreen forests, 950 m, 18 May 1988, C. Renuka 4078 (holotype CAL).

19. Calamus lakshmanae Renuka, J. Econ. Taxon. Bot. 14(3): 703. 1990. (Arecaceae)

Type: India, Karnataka, evergreen forests, 85 m, 14 March 1989, C. Renuka 4086 (holotype CAL).

20. Calamus prasinus Lakshmana \& Renuka, J. Econ. Taxon. Bot. 14(3): 705. 1990. (Arecaceae) 
Type: India, Karnataka, cane plantation, 35 m, 20 May 1988, C. Renuka 4082 (holotype CAL).

21. Calamus stoloniferus Renuka, J. Econ. Taxon. Bot. 14(3): 701. 1990. (Arecaceae)

Type: India, Karnataka, cane plantation, 85 m, 14 March 1989, C. Renuka 485 (holotype CAL).

22. Calanthe anjanae Lucksom, Indian J. Forest. 16(4): 386. 1994 (1993 publ. 1994) 'anjanii'. (Orchidaceae)

Type: India, Sikkim, Fimphu, 8th April 1990 and 20th April 1992, Lucksom 206a (holotype CAL).

23. Carex thanikaimoniana Govind., J. Econ. Taxon. Bot. 20(2): 305. 1996. (Cyperaceae)

Type: India, Tamil Nadu, Madurai district, Kodaikanal, Thanikaimoni 7082 (holotype CAL).

24. Cinnamomum assamicum S.C.Nath \& Baruah, J. Econ. Taxon. Bot. 25(1): 29. 2001. (Lauraceae)

Type: India, Assaam, Jorhat district, Puranimati, 86 m, S.C. Nath \& A. Baruah RRLJ 1861 (holotype CAL).

25. Croton bhasanthiae T.K.Paul, J. Econ. Taxon. Bot. 25(3): 594. 2002 (2001 publ. 2002). (Euphorbiaceae)

Type: India, West Bengal, Kalijhora, near Teesta river bank, ca. 304 m, 7.9.1999, T.K. Paul 26840A (holotype CAL).

26. Ecbolium ligustrinum (Vahl) Vollesen var. aryankavensis Remadevi \& Binojk., J. Econ. Taxon. Bot. 27(Suppl.): 1189. 2003. (Acanthaceae)

Type: India, Kerala, Quilon district, Aryankavu, 22.9.2001, S. Remadevi 325 (holotype MH).

27. Euphorbia katrajensis Gage var. kasaragodensis V.J.Nair, Binojk. \& R.Ansari, J. Econ. Taxon. Bot. 14(2): 471.1990 (Euphorbiaceae)

Type: India, Kerala, Cannanore district, Bela, 1.10.1982, R. Ansari 74431 (holotype CAL).

28. Fimbristylis cuneata Govind. \& S.K.Varma, J. Econ. Taxon. Bot. 21(2): 377. 1997. (Cyperaceae)

Type: India, Bihar, Sahibganj, Rajmahal Hills, Mirzachowki Rly. Station, Singh 5764 (holotype MH).

29. Fimbristylis diglumoides Govind. \& S.K.Varma, J. Econ. Taxon. Bot. 21(2): 377. 1997. (Cyperaceae)

Type: India, Bihar, Dumka, 25 km northeast of Antall village, Das 3542 (holotype CAL).

30. Fimbristylis hyalina Govind. \& Sasidh., J. Econ. Taxon. Bot. 21(2): 373. 1997. (Cyperaceae)

Type: India, Kerala, Thrissur, Charpa range, Athirapalli, Sasidharan 676 Hb. FRLHT, Bangalore (holotype $\mathrm{MH}$ ).

31. Fimbristylis perspicua Govind. \& Sasidh., J. Econ. Taxon. Bot. 21(2): 376. 1997. (Cyperaceae)

Type: India, Kerala, Thrissur, Peechi, N. Sasidharan 719 (holotype MH).

32. Gymnostachyum sahyadricum C.N.Mohanan, Remadevi \& Binojk., J. Econ. Taxon. Bot. 26(1): 38. 2002. (Acanthaceae)

Type: India, Kerala, Quilon district, Chokkampatty Hills, 23.3.1990, C.N. Mohanan 73469 (holotype $\mathrm{MH})$.

33. Hedychium satyanarayanum Sush.C.Srivast., Indian J. Forest. 22(1): 85. 1999. (Zingiberaceae)

Type: India, Meghalaya, Shillong, Old Barapani road, September 11th 1970, A.S. Rao 38640 (holotype ASSAM).

34. Helminthostachys zeylanica (L.) Hook. var. brachyspicae S.Nampy \& Madhus., J. Econ. Taxon. Bot. 18(1): 189. 1994. (Ophioglossaceae)

Type: India, Kerala, Malappuram district, Prakadavu, in open-waste lands, sea level, Santhosh Nampy \& A.K. Pradeep CU 44832 (holotype CAL).

35. Hybanthus vatsavayae C.S.Reddy, J. Econ. Taxon. Bot. 25(1): 219. 2001. (Violaceae) 
Type: India, Andhra Pradesh, Nalgonda district, Yadagirigutta, 18.10.1998, C.S. Reddy 1268 (holotype $\mathrm{MH}$ ).

36. Hygrophila salicifolia (Vahl) Nees var. cochinensis Remadevi \& Binojk., J. Econ. Taxon. Bot. 25(1): 233. 2001. (Acanthaceae)

Type: India, Kerala, Alleppey district, Arror, S. Remadevi 65 (holotype MH).

37. Kopsia majumdarii M.Gangop. \& Chakrab., J. Econ. Taxon. Bot. 16(1): 59. 1992. (Apocynaceae) Type: Malaya, Perak, open jungle, 300-600ft, Aug. 1885, King's Collector 7937 (holotype CAL).

38. Kyllinga eglandulosa Govind. \& K.Ramani, J. Econ. Taxon. Bot. 18(2): 335. 1994. (Cyperaceae)

Type: India, Karnataka, Bababudan hills, E. Govindarajalu 8726 (holotype CAL).

39. Kyllinga pluristaminea Govind. \& K.Ramani, J. Econ. Taxon. Bot. 18(2): 336. 1994. (Cyperaceae)

Type: India, Tamil Nadu, Madurai district, High Wavy Mts., Thuvanam, E. Govindarajalu C 239 (holotype CAL).

40. Liparis breviscapa A.P.Das \& Lama, J. Econ. Taxon. Bot. 16(1): 226. 1992. (Orchidaceae)

Type: India, West Bengal, Birch hill, Darjeeling, 2000 m, 4 August 1981, A.P. Das 603 (holotype CAL).

41. Millettia pseudoracemosa Thoth. \& S.Ravik., J. Econ. Taxon. Bot. 21(1): 239. 1997, 'pseudoracemosa'. (Leguminosae)

Type: India, Tamil Nadu, Anamalai Hills, Valparai, 350 m, 21.4.1995, S. Ravikumar 334A (holotype $\mathrm{MH})$.

42. Petalonema striato-theca R.K.Gupta, Indian J. Forest. 24(4): 500. 2001. (Scytonemataceae)

Type: India, Uttaranchal, Dehra Dun, 2298 m, 2.12.2000, R.K. Gupta 97904 (BSD: Cryptogamic Section, preserved in formalin solution).

43. Protasparagus biradarii Kamble, J. Econ. Taxon. Bot. 17(1): 197. 1993, 'biradari'. (Asparagaceae)

Type: India, Orissa, Mayurbhanj district, Dharamchampa, May 1941, Dr. Biswas's collector R.K. Kazi 88 (holotype CAL).

44. Protasparagus karthikeyanii Kamble, J. Econ. Taxon. Bot. 19(3): 735.1996 (1995 publ. 1996). (Asparagaceae)

Type: India, Maharashtra, Pune district, Madh in Junnar Taluka, June 1956, J.A. Vasavada 17049 (F. No. 2736) (holotype BSI).

45. Pycreus fasciculatus Govind., J. Econ. Taxon. Bot. 20(2): 301. 1996. (Cyperaceae)

Type: India, Kerala, Munar, Idimottai, E. Govindarajalu 15427 (holotype CAL).

46. Pycreus mahadevanii Govind., J. Econ. Taxon. Bot. 20(2): 299. 1996. (Cyperaceae)

Type: India, Karnataka, Shimoga, Barkana, E. Govindarajalu 5118 (holotype CAL).

47. Pycreus pyramidalis Govind., J. Econ. Taxon. Bot. 20(2): 301. 1996. (Cyperaceae)

Type: India, Tamil Nadu, Tirunelveli district, Courtallam, Shembagadevi-Honey Falls, E. Govindarajalu 10404 (holotype CAL).

48. Rhynchostylis albiflora I.Barua \& Bora, J. Econ. Taxon. Bot. 26(1): 251. 2002. (Orchidaceae)

Type: India, Assam, Jorhat (26 $47^{\prime} \mathrm{N}$ and $\left.94^{\circ} 12^{\prime} \mathrm{E}\right)$, Karanga, $86.8 \mathrm{~m}$ above MSL, 28.4.1994, Barua 3286 (holotype CAL).

49. Ruellia sivarajanii Sreedevi, Remadevi \& Binojk., J. Econ. Taxon. Bot. 27(Suppl.): 1186. 2003. (Acanthaceae)

Type: India, Kerala, Alappuzha, 15.12.2000, B. Sreedevi 168 (holotype CAL). 
50. Sansevieria maduraiensis Binojk., J. Econ. Taxon. Bot. 26(2): 458. 2002. (Dracaenaceae)

Type: India, Tamil Nadu, Madurai, 25.6.1993, Binojkumar s.n. (holotype CAL).

51. Sonerila inaequalis Murugan \& Manickam, J. Econ. Taxon. Bot. 25(3): 510. 2002 (2001 publ. 2002). (Melastomataceae)

Type: India, Tamil Nadu, Tirunelveli district, Courtallum hills, 1500-1700 m, 15.2.2000, C. Murugan XCH 18228 (holotype MH).

52. Trichodesma indicum (L.) Sm. var. betulense K.K.Khanna \& An.Kumar, Indian J. Forest. 24(2): 226. 2001. (Boraginaceae)

Type: India, Madhya Pradesh, Betul district, Ranipur, 21.4.1997, Anand Kumar 50178A (holotype CAL).

53. Trigonella emodi Benth. var. oblongifolia Balodi \& R.R.Rao, J. Econ. Taxon. Bot. 15(1): 188. 1991. (Fabaceae)

Type: India, Uttar Pradesh, Garhwal, near Ghorpatta, 2800 m, 23.8.1963, U.C. Bhattacharyya 29675A (holotype CAL).

54. Zeuxine dhanikariana Maina, Lalitha \& Sreek., J. Econ. Taxon. Bot. 25(1): 21. 2001. (Orchidaceae)

Type: India, Andaman and Nicobar Islnds, South Andaman, near Dhanikari, Goal Tikkiri, 10.4.1997, Maina \& Sreekumar 18433A (holotype CAL).

\section{CONCLUSION}

Essentially application of names of taxonomic groups is determined by means of nomenclatural types (Principle II of ICN, McNeill et al., 2012). The requirements for publication of names of new taxa are detailed in Articles 38-40 (McNeill et al., 2012). During the present analysis 34 holotypes at CAL, 13 at $\mathrm{MH}$, five at ASSAM, one each at BSI and BSD could not be traced in the respective herbarium. In two of our earlier publications (Bandyopadhyay et al., 2016, 2017) as well we have clearly indicated the missing of different types of many names that were published in two different journals, Rheedea (1991-2004) and Nordic Journal of Botany (1990-2004). Many fail to appreciate the importance of maintenance of types which help in resolving nomenclatural confusions that crop up in revisionary studies. The onus of depositing types goes with the author(s) while the maintenance responsibility goes with the herbarium concerned. Lapse either in deposition or maintenance leads to nonavailability of these specimens to bonafide researchers. Looking at the number of misplaced holotypes, it is necessary that we ensure the holotype deposition in a herbarium and its easy access to bonafide researchers prior to publication of new species. Herbaria which accept such depositions should ensure their scrupulous conservation.

During the 19th International Botanical Congress held at Shenzhen, China in July, 2017, the concept of registration of names of algae, bryophytes and vascular plants has been accepted, but it would come into effect after the 20th International Botanical Congress to be held at Rio de Janeiro, Brazil in 2023. It is beleieved that the problem of non-deposition of type specimens can be fully overcome when only the registration of names becomes mandatory.

\section{ACKNOWLEDGEMENTS}

We thank Dr. Paramjit Singh, Director, Botanical Survey of India (BSI), for approving this study as project work. We also thank Dr. S.K. Srivastava (Rtd.) (NRC, Dehra Dun); Dr. G.V.S. Murthy (Rtd.) and Dr. J.V. Sudhakar (SRC, Coimbatore); Dr. B.K. Sinha (ERC, Shillong); Dr. C. Murugan (ANRC, Port Blair) and Dr. J. Jayanthi (WRC, Pune), for facilitating the information on the type specimens in their respective herbarium. Dr. W. Arisdason (BSI) helped to improve the manuscript.

\section{REFERENCES}

[1] Bandyopadhyay S., Krishna G. and Venu P. Names of new taxa published and types deposited A case study. Annals of Plant Sciences 5.10: 1451-1457. 2016.

[2] Bandyopadhyay S., Krishna G. and Venu P. Names of new taxa published and types deposited A second case study. Annals of Plant Sciences 6.03: 1585-1589. 2017. 
[3] McNeill J.M., Barrie F.R., Buck W.R., Demoulin V., Greuter W., Hawksworth D.L., Herendeen P.S., Knapp S., Marhold K., Prado J., Prud'homme van Reine W.F., Smith G.F., Wiersema J.H. and Turland N.J. (Eds.) International Code of Nomenclature for algae, fungi, and plants (Melbourne Code). Regnum Vegetabile 154. Königstein: Koeltz Scientific Books, 2012.

Citation: S. Bandyopadhyay et al. , "Missing Holotypes of Names in Plants, Fungi and Algae Published From India", International Journal of Advanced Research in Botany, vol. 3, no. 3, p. 34-39, 2017. http://dx.doi.org/10.20431/2455-4316.0303005

Copyright: (C) 2017 Authors. This is an open-access article distributed under the terms of the Creative Commons Attribution License, which permits unrestricted use, distribution, and reproduction in any medium, provided the original author and source are credited. 